\title{
Delirium is not associated with anticholinergic burden or polypharmacy in older patients on admission to an acute hospital: an observational case control study
}

Hannah C. Moorey ${ }^{1,2}$, Sebastian Zaidman² and Thomas A. Jackson ${ }^{1,3^{*}}$

\begin{abstract}
Background: Older people are commonly prescribed multiple medications, including medications with anticholinergic effects. Polypharmacy and anticholinergic medications may be risk factors for the development of delirium.

Methods: Patients from a medical admission unit who were over 70, with DSM-IV diagnosed delirium and patients without delirium, were investigated. Number of drugs prescribed on admission and anticholinergic burden using two scales (the Anticholinergic Cognitive Burden Scale [ACB] and the Anticholinergic Drug Scale [ADS]) were recorded from electronic prescribing records. The relationship and predictive ability of these were explored.

Results: The sample included 125 patients with DSM-IV diagnosed delirium and 122 patients without delirium. The mean age of the sample was 84.0 years. The median number of drugs prescribed was 7: $79.8 \%$ were prescribed $\geq 5$ drugs and $29.0 \% \geq 10$ drugs. The median ACB score was 1 and the median ADS score was 1.5. $73.4 \%$ of patients had an ACB score of $\geq 1$ and $73.0 \%$ had a ADS score $\geq 1$. There was no association between: number of drugs prescribed, rate of polypharmacy, rate of excessive polypharmacy, ACB score and ADS score, and a diagnosis of delirium on admission. Only acetylcholinesterase inhibitor use predicted delirium (OR 3.86, $p=0.04)$ and the number of drugs prescribed was negatively correlated with age (spearman rho $=-0.18, p=0.006$ ).

Conclusion: Neither number of drugs prescribed, polypharmacy or anticholinergic burden were associated with delirium on admission, questioning the clinical usefulness of anticholinergic drug scales. Further research is needed to unpick fully the relationship between, drugs, anticholinergic burden, age, and prevalent delirium in older patients and whether there is any role for these scales in clinical practice.
\end{abstract}

Keywords: Delirium, Anticholinergic, Polypharmacy, Acetylcholine, Aged, Risk factor

\footnotetext{
* Correspondence: T.Jackson@bham.ac.uk

${ }^{1}$ Institute of Inflammation and Ageing, University of Birmingham,

Birmingham, UK

${ }^{3}$ Department of Geritric Medicine, University Hospitals Birmingham, Queen

Elizabeth Hospital, Birmingham, UK

Full list of author information is available at the end of the article
} 


\section{Background}

Delirium is an acute neuropsychiatric condition characterised by deficits in attention and cognition [1]. Delirium is important because it is common among older people acutely admitted to hospital and is associated with greater mortality, in-hospital falls and new institutionalization [2]. The aetiology of delirium is often multifactorial, however studies have found drugs often account for a significant proportion [3, 4]. Polypharmacy has been indicated as a precipitating factor in the development of delirium [5-7], as has the addition of multiple drugs as an inpatient [8]. Furthermore, the use of specific medications or medication classes have been identified as risk factors for the development of delirium [9]. Psychotropic drugs, particularly those with anticholinergic properties, have been implicated as precipitants [10].

Further investigation into the role of anticholinergic drugs in delirium is important as they are commonly prescribed medications [11], and they are biologically plausible as an aetiological factor in delirium [12]. Reduced cerebral acetylcholine has been hypothesized to be the common final pathway in the development of delirium in response to inflammation [13]. Delirium is associated with an increased risk of incident cognitive impairment [14] similar to that seen in chronic use of anticholinergic drugs [15]. This has led to the development of two methods to measure anticholinergic burden. First, anticholinergic burden can be estimated by measuring serum anticholinergic activity (SAA) in vitro. Second, expert-based anticholinergic drug scales estimate anticholinergic burden by assigning medications a score, based on high- low risk, which are then combined to produce an overall score for a patient. However, evidence of association between SAA and anticholinergic drug scales with delirium is contradictory [16-18]. Anticholinergic drug burden on admission has been associated with an admission with delirium [19] and incident delirium in patients admitted with acute stroke [20]. A rise in anticholinergic drug burden as an inpatient has also been associated with development of incident delirium in palliative patients [21]. However, other studies have found no association between the administration of anticholinergic medications and the development of incident delirium in older patients $[22,23]$, cancer patients [24] and intensive care patients [25].

Delirium is associated with poor outcomes and a high mortality rate [26] and early identification is important. Identifying patients at risk of delirium early may help improve detection and hence care, but it is not clear if medication identification has a role in this. Further research is needed to clarify which factors or scales are clinically useful in predicting delirium. Therefore, the aim of this study was to determine if anticholinergic drug burden, as measured by simple scales, or polypharmacy, were associated with delirium on admission to hospital.

\section{Method}

The objectives of this study were first to investigate associations between anticholinergic burden, measured through two different drug scales, and the presence of delirium in older people presenting to an acute hospital admission unit. Second, to investigate associations between the number of drugs prescribed pre-admission and the presence of polypharmacy with prevalent delirium in older people presenting to an acute hospital admission unit.

\section{Study sample}

Patients aged 70 years and over, with an unplanned medical admission to a United Kingdom university hospital between March 2013 and November 2014, were screened for delirium. A diagnosis of delirium that met the Diagnostic and Statistical Manual of Mental Disorders (4th ed., text rev; DSM-IV-TR; American Psychiatric Association, 2000) criteria was then made. This utilized screening with the Confusion Assessment Method (CAM) [27], Abbreviated Mental Test Score (AMTS) [28], the Digit Span test, and a detailed review of the medical notes. An expert geriatrician (TAJ) completed all screening. Potential participants who were unable to communicate because of severe sensory impairment or inability to speak in English were excluded, as were those deemed to be at risk of imminent death.

Participants with delirium were recruited as part of a larger study examining undiagnosed cognitive impairment in people with delirium [29]. Routinely collected data on a matched number of people screened as not having delirium were also collected. These were age, gender and hospital length of stay.

\section{Drug data collection}

Electronic prescribing records were used to collect admission medications and drug reconciliation is completed by pharmacists on the admissions ward to ensure accuracy of admission drug history. Drugs prescribed within $48 \mathrm{~h}$ of admission were recorded as pre-admission drugs. Clinical judgement was used to exclude drugs that were likely to be an acute prescription on that admission; by considering the likely indication, route of administration and whether drugs were prescribed on discharge. Both regular and PRN medications were included. Vitamins, topical preparations and optic preparations were included. Nutritional supplements were excluded. Any herbal, complimentary and alternative medicines, or over the counter medications regularly taken by the patient were only included if they were prescribed on admission to hospital. Polypharmacy was defined as 
5 or more drugs and excessive polypharmacy as 10 or more drugs. The Anticholinergic Cognitive Burden Scale (ACB) and the Anticholinergic Drug Scale (ADS) was then calculated for each participant based on the recorded preadmission drugs. The ACB and ADS scores were chosen as they are the most widely available scales. The DBI scale is another available scale, this was not used as it is a less specific measure of anticholinergic activity as it includes all psychotropic drugs.

\section{Statistical analysis}

Data were analysed using IBM SPSS Statistics for Windows (Version 22.0. Armonk, NY: IBM Corp.). Normality of data was assessed using the Shapiro-Wilk test. Differences in demographics, length of stay, number of drugs, polypharmacy, excessive polypharmacy and ACB and ADS scores between the delirium and no-delirium groups was assessed using an unpaired T-test, the independent samples MannWhitney $U$ test, or Chi Squared test as appropriate. Spearman Rank Coefficient was used to assess correlations between continuous variables. Logistic regression was used to generate odds ratios and $95 \%$ confidence intervals with: number of drugs, ACB score, ADS score, anticholinesterase inhibitor use, and polypharmacy, as predictor variables controlling for age as a recognized predictor of delirium. The significance level was set at $<0.05$.

\section{Results}

Of 1327 patients screened for delirium 228 were diagnosed with delirium. Of the patients without delirium data were collected for 125 patients to form the nodelirium group. Of the 228 diagnosed with delirium 125 were recruited and they formed the delirium group. Four patients from the no-delirium group were excluded as they were already included in the delirium group during a previous admission, giving a total sample of 251 (flow chart available as Additional file 1). The mean age of the sample was 84.0 ( $\mathrm{SD} \pm 6.60$ ) years. There was no significant difference in age or gender between the two groups. The median length of stay was 10 days (IQR 3-20). Participants in the delirium group stayed in hospital significantly longer than patients in the nodelirium group (15 vs 6 days respectively, $p<0.001$ ).

The median number of drugs prescribed was 7 (IQR 5-10). Polypharmacy was present in $79.8 \%$ of the sample and excessive polypharmacy was present in $29.0 \%$. There was no difference between those with and without delirium in number of drugs prescribed, or proportion of those with polypharmacy.

The median ADS score was 1.5 (IQR 0-3) and the median ACB was 1 (IQR 0-2). The median ACB score was the same in the delirium and no-delirium group. The median ADS score was slightly higher in the no-delirium group, 2, than in the delirium group, 1, but this was not significant $(p=0.09)$ (Table 1$)$.

The number of prescribed drugs was negatively correlated with age (spearman rho $=-0.176, p=0.006$ ). Acetlycholinesterase inhibitor prescription predicted delirium on admission when controlling for age (OR 3.86, $95 \%$ CI 1.05-14.22, $p=0.04$ ). Age, number of drugs, presence of polypharmacy or excessive polypharmacy, ADS and ACB scores did not predict delirium on admission.

\section{Discussion}

This observational case control study has demonstrated no relationship between anticholinergic burden and polypharmacy, with delirium in older people admitted to an acute hospital. Increasing age is actually associated with reduced number of drugs taken, and only taking anticholinesterase inhibitor drugs are associated with delirium. As expected the majority of older patients in this study were taking a large number of medications,

Table 1 Comparing Demographics, Anticholinergic Burden and Polypharmacy between Delirium and No-delirium Groups

\begin{tabular}{lllll}
\hline & Study Population $(n=251)$ & Delirium $(n=125)$ & No-delirium $(n=122)$ & Significance \\
\hline Mean Age (years, SD) & $83.97( \pm 6.60)$ & $84.4( \pm 6.52)$ & $83.54( \pm 6.67)$ & $p=0.21$ \\
Female (\%) & 67.3 & 62.4 & 72.4 & $p=0.95$ \\
Median Length of Stay (days, IQR) & $10(3-20)$ & $15(6-29)$ & $6(2-13)$ & $p<0.001$ \\
Median drugs prescribed & $7.00(5-10)$ & $7.00(5-10)$ & $7.00(5-10)$ & $p=0.72$ \\
Polypharmacy (\%) & 79.8 & 79.2 & 80.5 & $p=0.80$ \\
Excessive Polypharmacy (\%) & 29.0 & 28.8 & 29.3 & $p=0.94$ \\
Median ACB score & $1.00(0-2)$ & $1.00(0-3)$ & $1.00(1-2)$ & $p=0.58$ \\
ACB score $\geq 1$ (\%) & 73.4 & 68.8 & 78.0 & $p=0.10$ \\
Median ADS score & $1.50(0-3)$ & $1.00(0-2.5)$ & $2.00(1-3)$ & $p=0.09$ \\
ADS score $\geq 1$ (\%) & 73.0 & 67.2 & 78.9 & $p=0.39$ \\
AChE Inhibitor prescribed (\%) & 5.6 & 8.8 & 2.4 & $p=0.030$ \\
\hline
\end{tabular}


median 7 drugs, and $73 \%$ were exposed to at least one medication with anticholinergic effects. We found no relationship between either of the anticholinergic drug scales used in this study, ACB and ADS, and prevalent delirium on admission to hospital. We also found no association between the number of drugs patients were prescribed, or the presence of polypharmacy and prevalent delirium. Use of Acetylcholinesterase inhibitors was associated with delirium. Delirium is more common in patients with dementia [3] and this likely explains the correlation. However, acetylcholinesterase use is a poor proxy measure for dementia. Polypharmacy was negatively correlated with age in this population.

Strengths of the study include robust screening for delirium by an expert against recognized criteria, and the use of electronic records to accurately record admission drugs. Recognized limitations are that the drug history was taken in the acute setting which have been shown to be unreliable [30], but the drug reconciliation by ward based pharmacists, and the retrospective collection of the drug history from the electronic record, should have limited this potential bias. We did also recognize that drug prescription is not a reliable indicator of patient compliance. A further limitation is that due to study design we were not able to compare frailty or co-morbidity between those with and without delirium. Given the convenience nature of the no-delirium group it was not possible to be confident about how representative this group was of all patients without delirium.

This study supports previous work which has shown no association between anticholinergic burden and delirium [22-25]. However other studies have reported an association $[19,20]$. Studies have investigated different patient populations and this may in part explain the contrasting results. However, studies with a similar population to this study, older patients admitted to hospital, have also had mixed results $[19,22,23]$. The populations investigated were similar with regards to age. However, the cohort in the current study demonstrates a higher burden of polypharmacy, suggesting the cohort may be frailer and more multi-morbid, and perhaps of greater representation of real world practice.

Contrasting results may also be explained by limitations to the drug scales themselves. First, a number of drugs scales have been developed and there are variations between them. The ACB and ADS scales were used in this study. The only study to investigate a similar patient population and find positive results was by Best et al. [19] and used the Drug Burden Index (DBI) [31]. DBI is currently the only scale to consider drug dose but is also a broader drug scale including other sedative drugs without anticholinergic effects [31]. These differences may explain the contrast in the results.
Second, use of anticholinergic medications is likely to vary between populations and this may be reflected in the development of anticholinergic drug scales [32]. The use of scales in different populations may, therefore, not accurately assess anticholinergic burden. Furthermore, drug scales need to be regularly updated to include any new medications with anticholinergic effects [32]. Third, drug scales have been broadly criticised for oversimplifying a complicated pharmacological issue. For example, all scales use a simple linear additive model, however the risk of anticholinergic effects may not increase in this manner [33]. Also drug scales do not consider underlying patient characteristics including differences in pharmacodynamics, cholinergic reserve and endogenous anticholinergic activity [33]. These characteristics are likely to be even more important in a population of older patients, as investigated in this study.

This study shows a negative correlation between age and the number of drugs prescribed, which is in keeping with a previous report [34]. This may be explained by first, that the oldest old (age $\geq 85$ ) may be healthier than those who die earlier, and therefore take less medications; a survivor effect. Second, this may be due to deprescribing in the oldest old as medications are deemed clinically unnecessary towards the end of life.

This study raises doubts about the clinical utility of polypharmacy and anticholinergic drug scales, at predicting delirium in older hospital populations. However, further research is needed to clarify this and to investigate other factors, such as frailty, that may predispose some older people with polypharmacy and high anticholinergic drug use to delirium. Further research could also investigate which specific anticholinergic drug scale is most useful in delirium or the development of new scales with fewer limitations. The relationship between delirium, anticholinergic drug scale scores and acetylcholine levels or serum anticholinergic levels in vitro could also be investigated further. This may be particularly important in older people who are more likely to have decreased cholinergic reserve and more variable pharmacodynamics.

\section{Conclusion}

Neither number of drugs prescribed, polypharmacy nor anticholinergic burden were associated with delirium on admission. This study contributes to an existing volume of research which questions the usefulness of anticholinergic drug scales in predicting delirium. Further research is needed to fully unpick the relationship between drugs, anticholinergic burden and prevalent delirium in older patients, with a focus on additional factors which contribute to patient vulnerability to delirium and its complex aetiology. 


\section{Additional file}

Additional file 1: Figure S1. Flowchart of selection of study participants. Flowchart demonstrating selection of participants to the delirium and no-delirium groups and the number of patients recruited. The number of patients excluded and not recruited are also displayed and the reason they were not included. (DOCX $30 \mathrm{~kb}$ )

\section{Abbreviations}

ACB: The Anticholinergic Cognitive Burden Scale; AChEi: Acetylcholinesterase inhibitors; ADS: The Anticholinergic Drug Scale; AMTS: Abbreviated Mental Test Score; CAM: Confusion Assessment Method; DBI: Drug Burden Index; DSMIV: Diagnostic and Statistical Manual of Mental Disorders, 4th Edition; IQR: Interquartile range; SAA: Serum anticholinergic activity; SD: Standard deviation

\section{Acknowledgements}

We gratefully acknowledge the participants, carers and consultees who took part in the study.

\section{Funding}

TAJ was supported jointly by the Research into Ageing Fund; a fund set up and managed by Age UK and the British Geriatrics Society (\#367).

\section{Availability of data and materials}

Available on-line at the project page [35].

\section{Authors' contributions}

TAJ and HCM wrote the protocol. TAJ acquired subjects. HCM and SZ collected the data from the electronic notes. TAJ and HCM contributed equally to the first draft and all authors contributed to analysis and interpretation of data and revision of the manuscript and provided final edits. TAJ is guarantor. All authors read and approved the final manuscript.

\section{Competing interests}

The authors declare that they have no competing interests.

\section{Consent for publication}

Not applicable.

\section{Ethics approval and consent to participate}

Participants with delirium were part of a larger study examining undiagnosed cognitive impairment in people with delirium [29]. The study was approved by the National Research Ethics Service Committee of Yorkshire and Humberside (Ref: 12/YH/0534) and consent, or consultee declaration, was obtained from all participants who were diagnosed with delirium.

\section{Author details}

${ }^{1}$ Institute of Inflammation and Ageing, University of Birmingham, Birmingham, UK. ${ }^{2}$ College of Medical and Dental Sciences, University of Birmingham, Birmingham, UK. ${ }^{3}$ Department of Geritric Medicine, University Hospitals Birmingham, Queen Elizabeth Hospital, Birmingham, UK.

\section{Received: 27 May 2016 Accepted: 13 September 2016}

\section{Published online: 21 September 2016}

\section{References}

1. Inouye SK, Westendorp RGJ, Saczynski JS. Delirium in elderly people. Lancet 2014:383:911-22

2. Pendlebury ST, Lovett NG, Smith SC, Dutta N, Bendon C, Lloyd-Lavery A, et al. Observational, longitudinal study of delirium in consecutive unselected acute medical admissions: age-specific rates and associated factors, mortality and re-admission. BMJ Open. 2015:5.

3. George J, Bleasdale S, Singleton SJ. Causes and prognosis of delirium in elderly patients admitted to a district general hospital. Age Ageing. 1997;26:423-7.

4. Laurila JV, Laakkonen M-L, Timo SE, Pitkala KH. Predisposing and precipitating factors for delirium in a frail geriatric population. J Psychosom Res. 2008:65:249-54.
5. Kennedy M, Enander RA, Tadiri SP, Wolfe RE, Shapiro NI, Marcantonio ER. Delirium Risk Prediction, Healthcare Use and Mortality of Elderly Adults in the Emergency Department. J Am Geriatr Soc. 2014;62:462-9.

6. Martin NJ, Stones MJ, Young JE, Bédard M. Development of Delirium: A Prospective Cohort Study in a Community Hospital. Int Psychogeriatrics. 2000;12:117-27.

7. Hein C, Forgues A, Piau A, Sommet A, Vellas B, Nourhashémi F. Impact of polypharmacy on occurrence of delirium in elderly emergency patients. J Am Med Dir Assoc. 2014;15:850. e11-850. e15.

8. Inouye SK. Predisposing and precipitating factors for delirium in hospitalized older patients. Dement Geriatr Cogn Disord. 1999;10:393.

9. Clegg A, Young JB. Which medications to avoid in people at risk of delirium: a systematic review. Age Ageing. 2011;40:23-9.

10. Young J, Inouye SK. Delirium in older people. BMJ. 2007;334:842-6.

11. Sumukadas D, McMurdo MET, Mangoni AA, Guthrie B. Temporal trends in anticholinergic medication prescription in older people: repeated cross-sectional analysis of population prescribing data. Age Ageing. 2014; $43: 515-21$

12. Hshieh TT, Fong TG, Marcantonio ER, Inouye SK. Cholinergic deficiency hypothesis in delirium: a synthesis of current evidence. Journals Gerontol A, Biol Sci Med Sci. 2008:63:764-72.

13. van Gool WA, van de Beek D, Eikelenboom P. Systemic infection and delirium: when cytokines and acetylcholine collide. Lancet. 2010;375:773-5.

14. Davis DHJ, Muniz Terrera G, Keage H, Rahkonen T, Oinas M, Matthews FE, et al. Delirium is a strong risk factor for dementia in the oldest-old: a populationbased cohort study. Brain. Oxford University Press; 2012;135:2809-16.

15. Gray SL, Anderson ML, Dublin S, Hanlon JT, Hubbard R, Walker R, et al. Cumulative Use of Strong Anticholinergic Medications and Incident Dementia. JAMA Intern Med. 2015;175:401-7.

16. Flacker JM, Cummings V, Mach JR, Bettin K, Kiely DK, Wei J. The association of serum anticholinergic activity with delirium in elderly medical patients. Am J Geriatr Psychiatry. 1999:6:31-41.

17. Thomas C. Serum anticholinergic activity and cerebral cholinergic dysfunction: an EEG study in frail elderly with and without delirium. BMC Neurosci. 2008;9:86-7.

18. Watne LO, Hall RJ, Molden E, Ræder J, Frihagen F, Maclullich AMJ, et al, Anticholinergic Activity in Cerebrospinal Fluid and Serum in Individuals with Hip Fracture with and without Delirium. J Am Geriatr Soc. 2014;62(1):94-102.

19. Best O, Gnjidic D, Hilmer SN, Naganathan V, McLachlan AJ. Investigating polypharmacy and drug burden index in hospitalised older people. Intern Med J. 2013:43:912-8.

20. Caeiro L, Ferro JM, Claro MI, Coelho J, Albuquerque R, Figueira ML. Delirium in acute stroke: a preliminary study of the role of anticholinergic medications. Eur J Neurol. 2004;11:699-704.

21. Zimmerman KM, Salow M, Skarf LM, Kostas T, Paquin A, Simone MJ, et al. Increasing anticholinergic burden and delirium in palliative care inpatients. Palliat Med. 2014:28:335-41.

22. Luukkanen MJ, Uusvaara J, Laurila JV, Strandberg TE, Raivio MM, Tilvis RS, et al. Anticholinergic drugs and their effects on delirium and mortality in the elderly. Dement Geriatr Cogn Dis Extra. 2011;1:43-50.

23. Campbell N, Perkins A, Hui S, Khan B, Boustani M. Association Between Prescribing of Anticholinergic Medications and Incident Delirium: A Cohort Study. J Am Geriatr Soc. 2011:59:S277-81.

24. Gaudreau JD, Gagnon P, Harel F, Roy MA, Tremblay A. Psychoactive medications and risk of delirium in hospitalized cancer patients. J Clin Oncol. 2005;23:6712-8.

25. Pandharipande P, Shintani A, Peterson J, Pun BT, Wilkinson GR, Dittus RS, et al. Lorazepam is an independent risk factor for transitioning to delirium in intensive care unit patients. Anesthesiology. 2006;104:21-6.

26. Witlox J, Eurelings LSM, de Jonghe JFM, Kalisvaart KJ, Eikelenboom P, Van Gool WA. Delirium in elderly patients and the risk of postdischarge mortality, institutionalization, and dementia: a meta-analysis. JAMA. 2010:304:443-51.

27. Inouye SK, van Dyck CH, Alessi CA, Balkin S, Siegal AP, Horwitz RI. Clarifying Confusion: The Confusion Assessment MethodA New Method for Detection of Delirium. Ann Intern Med. 1990;113:941-8.

28. Hodkinson HM. Evaluation of a Mental Test Score for Assessment of Mental Impairment in the Elderly. Age Ageing. 1972;1:233-8

29. Jackson TA, MacLullich AMJ, Gladman JRF, Lord JM, Sheehan B. Diagnostic test accuracy of informant-based tools to diagnose dementia in older hospital patients with delirium: a prospective cohort study. Age Ageing 2016;45(4):505-11. 
30. Lau HS, Florax C, Porsius AJ, De Boer A. The completeness of medication histories in hospital medical records of patients admitted to general internal medicine wards. Br J Clin Pharmacol. 2000;49:597-603.

31. Hilmer SN, Mager DE, Simonsick EM, Cao Y, Ling SM, Windham BG, et al. A drug burden index to define the functional burden of medications in older people. Arch Intern Med. 2007;167:781-7.

32. Lertxundi U, Domingo-Echaburu S, Hernandez R, Peral J, Medrano J. Expertbased drug lists to measure anticholinergic burden: similar names, different results. Psychogeriatrics. 2013;13:17-24.

33. Kersten H, Wyller TB. Anticholinergic Drug Burden in Older People's Brain ? How well is it Measured? Basic Clin Pharmacol Toxicol. 2014;114:151-9.

34. Onder G, Liperoti R, Fialova D, Topinkova E, Tosato M, Danese P, et al. Polypharmacy in Nursing Home in Europe: Results From the SHELTER Study. J Gerontol Ser A Biol Sci Med Sci. 2012;67A:698-704.

35. Delirium in general hospital as a marker of undiagnosed dementia: evaluation of pragmatic methods for screening and follow-up - University of Birmingham [Internet]. [cited 2016 May 25]. Available from: http://www. birmingham.ac.uk/research/activity/mds/projects/ii/delhosp/index.aspx.

Submit your next manuscript to BioMed Central and we will help you at every step:

- We accept pre-submission inquiries

- Our selector tool helps you to find the most relevant journal

- We provide round the clock customer support

- Convenient online submission

- Thorough peer review

- Inclusion in PubMed and all major indexing services

- Maximum visibility for your research

Submit your manuscript at www.biomedcentral.com/submit
Biomed Central 